\title{
On the Width of the Last Scattering Surface
}

\author{
N. PIRES*, M. A. S. NOBRE ${ }^{\dagger}$, J. A. S. LIMA \\ Departamento de Física, \\ Universidade Federal do Rio Grande do Norte, C.P. 1641, 59072-970, Natal, RN, Brasil
}

(June 29, 2018)

\begin{abstract}
We discuss the physical effects of some accelerated world models on the width of the last scattering surface (LSS) of the cosmic microwave background radiation (CMBR). The models considered in our analysis are X-matter (XCDM) and a Chaplygin type gas. The redshift of the LSS does not depend on the kind of dark energy (if XCDM of Chaplygin). Further, for a Chaplygin gas, the width of the LSS is also only weakly dependent on the kind of scenario (if we have dark energy plus cold dark matter or the unified picture).
\end{abstract}

\section{INTRODUCTION}

It is widely known that the last scattering surface (LSS) has a finite thickness $\Delta z$ (in redshift space) because the hydrogen recombination process takes a finite time. This fact has important consequences for the physics relating theoretical predictions and observations of the the angular pattern of CMBR anisotropies. In particular, it implies that anisotropies

\footnotetext{
*npires@dfte.ufrn.br

†assunta@fisica.ufpb.br

‡limajas@astro.iag.usp.br
} 
at length scales smaller than $\Delta z$ must be naturally suppressed.

On the other hand, recent observations from type Ia Supernovae [1] combined with CMBR experiments [2] strongly suggest an accelerating and nearly flat Universe $\left(q_{o}<\right.$ $\left.0, \Omega_{\text {Total }} \approx 1\right)$. In the framework of general relativity both facts are readily accommodated by assuming the existence of an extra dark energy (DE) component with negative pressure (in addition to cold dark matter $(\mathrm{CDM})$ ). Besides the cosmological constant $[3](\Lambda)$ and a scalar field $[4](\Phi)$, there are at least 3 distinct dark energy candidates proposed in the literature, namely: vacuum decay $\Lambda(t)$ - models [5], X-matter [6], and a Chaplygin gas [7].

In this paper we discuss the width of the LSS for accelerating world models driven by the last two above quoted DE candidates (X-matter and Chaplygin gas). Since the dark energy component present in these models is separately conserved, the consequences to LSS are completely different to what happens with accelerating models endowed with any kind of adiabatic photon creation [5,8]. With basis on the WMAP observations [9], in the present calculations we consider $H_{0}=71 \mathrm{~km} \mathrm{~s}^{-1} \mathrm{Mpc}^{-1}, \Omega_{b}=0.04$ for baryonic matter, and $\Omega_{D M}=0.27$ for cold dark matter.

\section{LAST SCATTERING SURFACE AND THE RECOMBINATION PROCESS}

The probability that a CMBR photon undergone its last scattering between $z$ and $z+d z$ is

$P=1-e^{-\tau(z)}$, where $\tau=\int_{0}^{z} n_{e} \sigma_{T} c d t$ is the optical depth to redshift $z, \sigma_{T}$ is the Thomson cross section and $n_{e}$ is the density of electrons. The quantity $d P(z) / d z$ determines the visibility function, that is, the probability distribution for the redshifts where the CMBR photons had their last scattering. By defining the effective profile of the LSS: $V(z)=$ $d P(z) / d z=e^{-\tau(z)} d \tau / d z$, and fitting the visibility curve with a Gaussian form, the standard deviation yields a reasonable estimate of the LSS width whereas its peak stands for the beginning of the recombination epoch [10].

At least 3 physical process are acting during and after recombination on the baryonic matter: photoionization, cooling from recombination, and Compton cooling-heating. Hence, 
neglecting the helium and treating the hydrogen atom as a two-level system, the fraction $x_{e}$ of ionized matter obeys [11]

$$
\frac{d x_{e}}{d t}=\frac{\Lambda_{2 s, 1 s}}{\left(\Lambda_{2 s, 1 s}+\beta_{e}\right)}\left[\beta_{e} e^{-\frac{\left(B_{1}-B_{2}\right)}{k_{\beta} T_{\gamma}}}\left(1-x_{e}\right)-\frac{a_{r} \rho x_{e}^{2}}{m_{p}}\right],
$$

where $B_{1}=13.6 \mathrm{eV}$ is ground state energy, $B_{2}=3.4 \mathrm{eV}$ is the first excited state energy, $\beta_{e}=\left(2 \pi m_{e} k_{B} T_{\gamma}\right)^{3 / 2} h^{-3} e^{-\left(B_{2} / k_{B} T_{\gamma}\right)} a_{r}$ is the photoionization rate, $a_{r}=2.84 \times 10^{-11} T_{m}^{-1 / 2} \mathrm{~cm}^{3}$ $\mathrm{sec}^{-1}$ is the recombination coefficient, and $\Lambda_{2 s, 1 s}=8.272 \mathrm{sec}^{-1}$ is the two-photon emission rate. After decoupling, the matter temperature $\left(T_{m}\right)$ of the neutral atoms fall faster than the radiation temperature $\left(T_{\gamma}\right)$. The matter temperature decreasing is governed by the equation

$$
\frac{d T_{m}}{d t}=T_{m}\left[-2 \frac{\dot{R}}{R}-\frac{\dot{x}_{e}}{3\left(1+x_{e}\right)}\right]-\frac{8 \sigma_{T} b}{3 m_{e} c} \frac{T_{\gamma}^{4} x_{e}}{\left(1+x_{e}\right)}\left(T_{m}-T_{\gamma}\right),
$$

where $\dot{R} / R$ is the Hubble parameter, $\dot{x}_{e}=d x_{e} / d t$, and $8 \sigma_{T} b / 3 m_{e} c=8.02 \times 10^{-9} \mathrm{sec}^{-1} \mathrm{~K}^{-4}$.

\section{VISIBILITY FUNCTION: MAIN RESULTS}

Let us now discuss the visibility function for the accelerating models (X-matter and Chaplygin gas) quoted in the introduction.

\section{(i) X-Matter Models:}

In cosmological scenarios driven by X-matter plus cold dark matter (sometimes called XCDM parametrization) both fluid components are separately conserved. The equation of state of the dark energy component is $p_{x}=w(z) \rho_{x}$. Unlike to what happens with scalar field motivated models where $w(z)$ is derived from the field description, the expression of $w(z)$ for XCDM scenarios must be assumed a priori. Models with constant $w$ are the simplest ones and their free parameters can easily be constrained from the main cosmological tests. In what follows we focus our attention to this class of models assuming a flat geometry. The differential time-redshift relation is

$$
d t=\frac{1}{H_{0}} \frac{d x}{x\left[\Omega_{M} x^{-3}+\left(1-\Omega_{M}\right) x^{-3(1+\omega)}\right]^{1 / 2}},
$$


where $\Omega_{M}=1-\Omega_{X}$ is the density parameter of the dark matter. Taking the limiting case $\omega=-1$, the $\Lambda$ CDM results are recovered. The basic results are presented in Table 1 . The left panel of Figure 1 shows the corresponding visibility function.

(ii) Chaplygin Gas:

This class of accelerating models refers to an exotic fluid whose equation of state is given by $p_{C}=-A / \rho_{C}^{\alpha}$, where $A$ and $\alpha$ are positive parameters. Actually, the above equation for $\alpha \neq 1$ generalizes the original Chaplygin equation of state whereas for $\alpha=0$, the model behaves like scenarios with cold dark matter plus a cosmological constant $(\Lambda \mathrm{CDM})$. The dynamics of such a fluid is similar to non-relativistic matter (dark matter) at high redshift and as a negative-pressure DE component at late times. Two different pictures are usually considered in the literature: the first is a flat scenario driven by a non-relativistic matter plus the Chaplygin gas as a dark energy (GCgCDM), whereas in the second one, the Chaplygin type gas together with the observed baryonic content are responsible by the dynamics of the present-day universe (unifying dark matter with dark energy (UDME or Quartessence). The differential age-redshift relation as a function of the observable now reads

$$
d t=\frac{1}{H_{0}}\left\{\frac{x}{\Omega_{j}+\left(1-\Omega_{j}\right) x^{3}\left[A_{s}+\left(1-A_{s}\right) x^{-3(\alpha+1)}\right]^{\frac{1}{1+\alpha}}}\right\}^{1 / 2} d x,
$$

where $A_{s}=A / \rho_{c_{0}}^{1+\alpha}$ and $H_{0}$ is the Hubble constant. $\Omega_{j}$ stands for baryonic + dark matter density parameter in GCgCDM models but only to the baryonic matter density parameter in the UDME (Quartessence) scenarios. The results are presented in Table 2 and the right panel of Figure 1 show the corresponding visibility function to both cases.

In summ, the thickness of the LSS has been discussed using two different accelerating world models. Tables 1 and 2 show the main conclusions of this work. As we have seen, the X-matter models present the same behaviour of constant $\Lambda$ models, regardless of the value of $\omega$. Probably, more important, the recombination epoch is just the same for all models driven by X-matter and the Chaplygin gas (it is located at redshift $z_{\text {rec }}=1.127$ ). Further, the width of the LSS is only weakly dependent on the kind of dark energy models considered here. As one can see from Table 2, the UDME models (in which the C-gas plays the role of 
both dark matter and dark energy) has a little influence on the width of the LSS. This is in line with the visibility function presented in figure (b).

Finally, for the sake of comparison, we have also computed $z_{r e c}$ and the width of the LSS for models with decaying vacuum energy density [5] and adiabatic gravitational creation of matter and radiation [8]. Due to the adiabatic creation of photons, the results concerning the width of the LSS and $z_{\text {rec }}$ are strongly modified. This means that the physics of the LSS may constrain with great accuracy any model endowed with photon creation because the temperature law of the CMBR is modified. This problem will be discussed in a forthcoming communication.

Acknowledgements:The authors are grateful to J. C. Neves de Araujo by the numerical code and many helpful discussions. 


\section{REFERENCES}

[1] S. Permultter et al., Nature 391, 51 (1998).

[2] P. de Bernardis et al., Nature 404, 955 (2000).

[3] T. Padmanabhan, Phys. Rep. 380, 235 (2003); P. J. E. Peebles and B. Ratra, Rev. Mod. Phys. 75, 559 (2003).

[4] R. R. Caldwell, Braz. J. Phys. 30, 215 (2000).

[5] M. Özer and M. O. Taha Phys. Lett. B171, 363 (1986); J. C. Carvalho et al., Phys. Rev. D46 2404 (1992); J. A. S. Lima and J. M. F. Maia, Phys. Rev D49, 5597 (1994); J. M. Overduin and F. I. Cooperstock, Phys. Rev. D58, 043506 (1998); J. A. S. Lima, Braz. J. Phys. 34, 194 (2004).

[6] M. S. Turner and M. White, Phys. Rev. D56, R4439 (1997); T. Chiba, N. Sugiyama and T. Nakamura, Mon. Not. Roy. Astron. Soc. 289, L5 (1997); J. S. Alcaniz and J. A. S. Lima, ApJ 521, L87 (1999); ApJ 550, L133 (2001); S. A. Bludman and M. Roos, Phys. Rev. D 65, 043503 (2002).

[7] A. Kamenshchik et al., Phys. Lett. B511, 265 (2001); N. Bilic et al., Phys. lett. B535 17 (2002); M. C. Bento et al., Phys. Rev. D66, 043507 (2002); A. Dev et al., Phys. Rev D67, 023515 (2003); M. Makler et al., Phys. Lett. B555, 1 (2003).

[8] Prigogine et al. Gen, Rel Grav. 21, 767 (1989); see also, M. O. Calvão, J. A. S. Lima and I. Waga, Phys. Lett. A162, 223 (1992); J. A. S. Lima and A. S. M. Germano Phys. Lett. A 170, 373 (1992); J. A. S. Lima and J. S. Alcaniz, A\&A 348, 1 (1999); L. P. Chimento, A. S. Jakubi and N. A. Zuccala, Phys. Rev. D63, 103508 (2001); W. Zimdahl, D. J. Schwarz, A. B. Balakin and D. Pavón, Phys. Rev. D64, 063501 (2001); M. P. Freaza, R. S. de Souza and I. Waga, Phys. Rev. D66, 103502 (2002).

[9] D. M. Spergel et al., Ap. J. Suppl. 148, 175 (2003). 
[10] B. J. T. Jones and R. F. G. Wyse Astron. \& Astrophys. 149, 144 (1985)

[11] P. J. E. Peebles, Principles of Physical Cosmology (Princeton University Press, Princeton, 1993) 


\section{TABLES}

Redshift and width of the LSS for X-matter models

\begin{tabular}{lccccccc}
\hline \hline MODEL & $\Omega_{M}$ & $\Omega_{x}$ & $\lambda$ & $\omega$ & $\Delta z$ & $z_{\text {rec }}$ & $\Delta \mathrm{l}(\mathrm{Mpc})$ \\
X-matter & 0.27 & 0.69 & - & 0.0 & 68.7 & 1127.4 & 13.8 \\
& 0.27 & 0.69 & - & -0.5 & 68.7 & 1127.4 & 13.8 \\
& 0.27 & 0.69 & - & -1.0 & 68.7 & 1127.4 & 13.8 \\
$\lambda$-model & 0.27 & - & 0.69 & - & 68.7 & 1127.4 & 13.8 \\
\hline \hline
\end{tabular}


Redshift and width of the LSS for a Chaplygin gas.

\begin{tabular}{|c|c|c|c|c|c|c|c|}
\hline MODEL & $\Omega_{j}$ & $\Omega_{C}$ & $\alpha$ & $A_{s}$ & $\Delta z$ & $z_{r e c}$ & $\Delta \mathrm{l}(\mathrm{Mpc})$ \\
\hline \multirow[t]{8}{*}{ GCgCDM } & 0.31 & 0.69 & 1.0 & 0.4 & 108.8 & 1127.4 & 13.2 \\
\hline & 0.31 & 0.69 & 1.0 & 0.5 & 68.7 & 1127.4 & 8.6 \\
\hline & 0.31 & 0.69 & 1.0 & 0.7 & 108.8 & 1127.4 & 14.6 \\
\hline & 0.31 & 0.69 & 1.0 & 0.9 & 108.8 & 1127.4 & 16.7 \\
\hline & 0.31 & 0.69 & - & 1.0 & 68.7 & 1127.4 & 13.8 \\
\hline & 0.31 & 0.69 & 0.8 & 0.5 & 108.8 & 1127.4 & 13.8 \\
\hline & 0.31 & 0.69 & 0.5 & 0.5 & 108.3 & 1127.4 & 14.0 \\
\hline & 0.31 & 0.69 & 0.2 & 0.5 & 108.3 & 1127.4 & 14.5 \\
\hline \multirow[t]{8}{*}{ UDME } & 0.04 & 0.96 & 1.0 & 0.4 & 108.8 & 1127.4 & 13.7 \\
\hline & 0.04 & 0.96 & 1.0 & 0.5 & 68.7 & 1127.4 & 10.7 \\
\hline & 0.04 & 0.96 & 1.0 & 0.7 & 108.8 & 1127.4 & 16.1 \\
\hline & 0.04 & 0.96 & 1.0 & 0.9 & 108.8 & 1127.4 & 20.7 \\
\hline & 0.04 & 0.96 & - & 1.0 & 68.7 & 1127.4 & 38.3 \\
\hline & 0.04 & 0.96 & 0.8 & 0.5 & 108.8 & 1127.4 & 14.6 \\
\hline & 0.04 & 0.96 & 0.5 & 0.5 & 108.8 & 1127.4 & 15.1 \\
\hline & 0.04 & 0.96 & 0.2 & 0.5 & 108.8 & 1127.4 & 16.0 \\
\hline
\end{tabular}




\section{FIGURES}
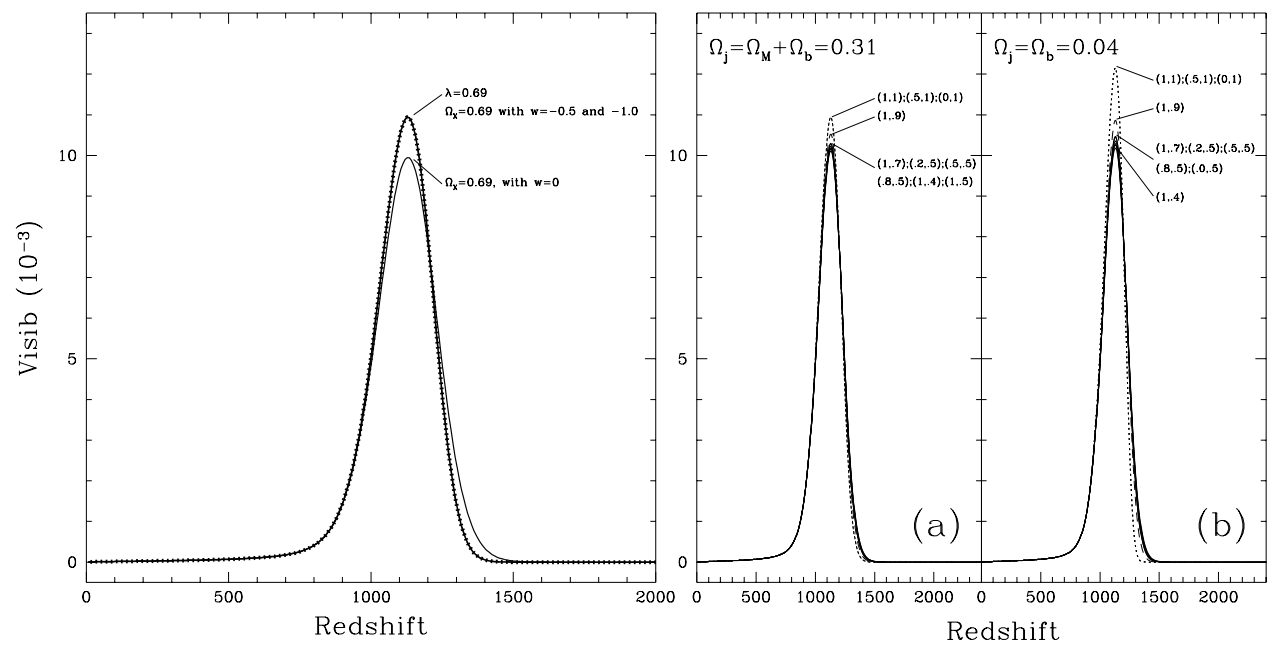

FIG. 1. Visibility function for models with X-matter and a Chaplygin gas. In the first panel (left), the dotted line is the prediction for $\Lambda \mathrm{CDM}$ models whereas the others lines are for XCDM. The two panels on the right show the results for a Chaplygin type gas: (a) models with CDM plus a Chaplygin gas (GCgCDM), and (b) Chaplygin gas in the unified (UDME) scenario. Note that the visibility function is not very affected by these models. 